\title{
Dinámica de sales en el distrito de riego La Doctrina, Colombia ${ }^{1}$
}

\author{
Salt dinamics in the irrigation district of \\ La Doctrina, Colombia
}

\author{
Teobaldis Mercado Fernández ${ }^{2,3}$; Manuel Ortega Escobar²; Abrahan Arenas Tawil ${ }^{3}$; \\ Enrique Combatt Caballero ${ }^{3}$
}

\begin{abstract}
RESUMEN
La presente investigación se realizó en el distrito de riego y drenaje La Doctrina y sus zonas aledañas con un área de 4160 hectáreas, localizado en el Departamento de Córdoba al Norte de Colombia, el cual posee una precipitación media de $1000 \mathrm{~mm}$, una temperatura de $28{ }^{\circ} \mathrm{C}$ y humedad relativa del $80 \%$. Se evaluó la dinámica de las sales en el suelo y los mantos freáticos someros a través de la medición de profundidades y cotas de niveles freáticos durante un año (junio de 2003 a junio 2004) y determinación de la salinidad de suelos y aguas freáticas en una red de 27 pozos de observación en tres fechas de muestreo (septiembre 2003, noviembre de 2003 y marzo de 2004). Se encontraron zonas con profundidad del nivel freático menor a $0,5 \mathrm{~m}$ y con salinidad mayor de $2 \mathrm{dS} / \mathrm{m}$, con variación temporal y espacial de las sales detectadas a partir de los planos de salinidad analizados, lo cual representa un alto riego de salinidad para estos suelos. Se observó que las zonas salinas coinciden con las zonas de descarga del flujo freático. Las principales sales solubles presentes fueron el cloruro de sodio $(\mathrm{NaCl})$ y el cloruro de magnesio $\left(\mathrm{MgCl}_{2}\right)$, las cuales son de alta solubilidad y toxicidad para las plantas.
\end{abstract}

Palabras clave: Isobatas, isohipsas, mapas de salinidad, cloruro de sodio, cloruro de magnesio.

\begin{abstract}
The present research was conducted in the irrigation and drainage district of La Doctrina and its adjacent zones with an area of 4160 hectares, located in the Department of Córdoba in the north of Colombia, which has an average precipitation of $1000 \mathrm{~mm}$, a temperature of $28^{\circ} \mathrm{C}$ and relative humidity of $80 \%$. The dynamics of salt in the soil and the superficial aquifers were evaluated through the measurement of depths and elevations of the water table during one year (June 2003 to June 2004) and the determination of the salinity of soils and aquifers in a chain of 27 observation wells on three sampling dates (September 2003, November 2003 and March 2004). Zones were found with a water table depth of less than $0.5 \mathrm{~m}$ and with salinity greater than $2 \mathrm{dS} / \mathrm{m}$, with temporal and spatial variation of salts detected from the planes of salinity analyzed, which represent high salinity irrigation for those soils. It was observed that the saline zones coincide with the zones of discharge of the phreatic flow. The principal soluble salts present were sodium chloride $(\mathrm{NaCl})$ and magnesium chloride $\left(\mathrm{MgCl}_{2}\right)$, which are of high solubility and toxicity for the plants.

Key words: Isobatas, isohypsas, salinity maps, sodium chloride, magnesium chloride.
\end{abstract}

\section{Introducción}

El manejo inadecuado de láminas de riego, precipitaciones excesivas y tipos de suelos son factores causantes de diferentes grados de problemas de drenaje y salinización en las zonas agrícolas, originando la degradación y pérdidas del potencial productivo de los suelos y afectando directamente el desarrollo de la agricultura intensiva. La elevación del manto freático causado por el riego, una proximidad a un río y las precipitaciones generan una acumulación de sales en el suelo (Tanji, 1990; Bustamante, 1993; Espinosa, 1995).

Los mapas de isobatas representan zonas con igual profundidad del nivel freático, estas se obtienen por la medición directa en los pozos de observación

1 Proyecto de investigación Universidad de Córdoba, Colombia - Colegio de Postgraduados, México.

2 Colegio de Postgraduados, Centro de Hidrociencias. Km 36.5 Carretera México - Texcoco, C.P. 56230. Telefax 525959520200 Extensión 1167. E-mail: teobaldis@gmail.com. Montecillo, Texcoco, Estado de México, México.

3 Facultad de Ciencias Agrícolas - Facultad de Ciencias Básicas e Ingenierías, Universidad de Córdoba, Carrera 6ª \# 76-103. Telefax 574 7860255. Montería, Córdoba, Colombia. 
desde la superficie del suelo, y determinan las zonas con riesgos de salinización y necesidad de drenaje en especial en períodos o etapas críticas, como cuando el cultivo es más sensible a los niveles freáticos someros (Pizarro, 1978; Tanji, 1990; Bustamante, 1993; CNA, 2007).

Los mapas de superficies freáticas o isohipsas representan zonas de igual cota de los niveles freáticos. La dirección del flujo subterráneo es normal a las isolíneas y de las líneas de mayor potencial a las de menor potencial, la densidad de flujo la define la pendiente entre las isohipsas, o sea la relación entre la diferencia de alturas y la distancia entre ellas.

Si en cada pozo de observación se toman muestras de agua freática y de suelo en áreas adyacentes y se realizan los análisis de salinidad, entonces es posible construir mapas de salinidad de aguas freáticas y suelos. Estos mapas son muy importantes para entender los procesos de migración y acumulación de sales en una zona. Las comparaciones de las composiciones de los iones disueltos en el agua freática guardan siempre una cierta relación con la composición de los iones en los suelos (Custodio y Llamas, 1983; Martínez, 1986; Hassan, 1994; Mercado, 1999).

La intensa y continua recarga originada por las filtraciones del agua de riego provoca la sobre elevación de los mantos freáticos en los distritos de riego de las zonas áridas y semiáridas. Cuando el manto freático invade la zona de raíces, se presentan problemas de drenaje y salinidad, afectando la producción de los cultivos y la productividad de los suelos. En ocasiones, los problemas de mantos freáticos elevados y salinidad son de tal magnitud que la actividad agrícola resulta ser incosteable. El drenaje agrícola subterráneo es una alternativa para la rehabilitación de los suelos con este tipo de problemas (Niazi et al., 2002; Timm et al., 2002; Lázaro et al., 2005; Yang, 2008).

En los problemas de drenaje subterráneo se debe tener en cuenta la profundidad mínima a la cual se debe ubicar el nivel freático para que no cause disminución en la producción de los cultivos y se genere una salinización del suelo, por lo que los esquemas de riego para el suministro de agua a los cultivos y control de la salinidad del suelo deben ser adecuados para un descenso de los niveles freáticos someros a fin de incrementar la producción. Una vez que se logra el descenso de los niveles a umbrales adecuados, la operación del drenaje necesita ser optimizada de acuerdo con la dinámica de las variaciones espacio-temporales (Hornbuckle et al., 2007; Rana et al., 2008; Ritzema et al., 2008).

Según Cenicaña (1991) y Cruz (1995), la producción de caña de azúcar se puede reducir hasta en 35 toneladas por hectárea, cuando el nivel freático se mantiene a una profundidad menor de $70 \mathrm{~cm}$. Si en los suelos se tiene una humedad excesiva durante la preparación del terreno, entonces las labores de siembra y cosecha sufren un retraso, además los suelos se compactan y el porcentaje de germinación se reduce, aumentando los costos por concepto de resiembras y, por consiguiente, los costos totales de producción. Pero niveles freáticos entre 1,0 y $1,2 \mathrm{~m}$ de profundidad son adecuados para el desarrollo y producción del cultivo de la caña de azúcar.

Estudios realizados en Córdoba, Argentina, usando el modelo de simulación del balance hídrico FREAT 1, en suelos Haplustol udorténtico y Natralbol típico, los dos con niveles freáticos oscilando a menos de tres metros de profundidad, mostraron que el modelo fue apropiado para predecir oscilaciones temporales del manto freático y evaluar el riesgo de inundación por anegamiento y además fue un excelente indicador del riesgo de salinización de los suelos (Degioanni et al., 2006).

En estudio de salinidad de los mantos freáticos someros realizados en los años 2002 y 2004 en el distrito de riego de la provincia de Mendoza, Argentina, se encontró que los planos de salinidad construidos con la base de datos depurada muestran los sectores afectados con los distintos intervalos de salinidad freática y su efecto en el desarrollo de los cultivos (Ortiz et al., 2005).

El objetivo de la presente investigación fue estudiar la dinámica de las sales en el suelo y aguas freáticas en la salinización progresiva de los suelos del distrito de riego La Doctrina, Departamento de Córdoba, Colombia.

\section{Metodología}

\section{Generalidades del área de estudio}

La zona de estudio se encuentra dentro de la región de planicie aluvial y marismas del nivel de base del río Sinú, Córdoba, Colombia y se ubica entre los meridianos $75^{\circ} 48^{\prime} 06^{\prime \prime}$ y $75^{\circ} 58^{\prime} 06^{\prime \prime}$ de longitud Oeste y los paralelos $9^{\circ} 17^{\prime} 58.5^{\prime \prime}$ y $9^{\circ}$ 25' 51" de latitud Norte. En su parte Sur, la zona se originó principalmente a partir de sedimentos 
fluviolacustres del cuaternario con granulometría diversa. En su extremo Septentrional presenta la influencia de marismas. El relieve es fundamentalmente plano, y en ciertos espacios es cóncavo, desenvolviéndose desde una fisiografía de terrazas bajas hasta la zona de marismas que dominan en su extremo Norte. La zona presenta una precipitación promedio $1000 \mathrm{~mm} / \mathrm{año}$, temperatura de $28{ }^{\circ} \mathrm{C}$ y humedad relativa de $80 \%$. El comportamiento de las lluvias es unimodal, con concentración de precipitaciones entre agosto y octubre, siendo este último el mes más lluvioso, en tanto que el mes más seco es marzo (Arenas et al., 2003).

En el área de estudio correspondiente, el distrito de riego y drenaje La Doctrina, Departamento de Córdoba, Colombia, con extensión de 4160 hectáreas, se construyeron 27 pozos de observación con su respectiva nivelación topográfica. En cada uno de los pozos se midió la profundidad y la cota de los mantos freáticos mensualmente desde junio de 2003 a junio de 2004, además se realizaron tres muestreos de suelos en los entornos de los pozos y de aguas freáticas para los respectivos análisis de salinidad en las siguientes fechas: septiembre de 2003, noviembre de 2003 y marzo de 2004.

Las muestras colectadas se enviaron al laboratorio de suelos y aguas de la Universidad de Córdoba, Colombia donde se realizaron los análisis de salinidad de acuerdo con las metodologías estandarizadas por la APHA (1995). Las determinaciones realizadas para aguas freáticas fueron. Conductividad eléctrica $\left(d \mathrm{Sm}^{-1}\right), \mathrm{pH}$, Cationes $\left(\mathrm{Ca}^{2+}, \mathrm{Mg}^{2+}, \mathrm{Na}^{+}, \mathrm{K}^{+}\right.$en $\left.\mathrm{mmol}_{c} \cdot \mathrm{L}^{-1}\right)$, Aniones $\left(\mathrm{Cl}^{-}, \mathrm{SO}_{4}^{2-}, \mathrm{CO}_{3}^{2-}, \mathrm{HCO}_{3}^{-}\right.$en $\left.\mathrm{mmol}_{c} \cdot L^{-1}\right)$ y concentración total de sales $\mathrm{mmol}_{c} \cdot L^{-1}$. En los análisis químicos de suelos se determinaron: Conductividad eléctrica en extracto de saturación $\left(d S m^{-1}\right), \mathrm{pH}$, cationes y aniones en solución del suelo $\left(\mathrm{mmol}_{c} \cdot \mathrm{L}^{-1}\right)$ e intercambiables $\left(\mathrm{cmol}_{c} \cdot \mathrm{Kg}^{-1}\right)$ y azufre $m g \cdot \mathrm{Kg}^{-1}$ (APHA, 1995).

Con la información de campo y de laboratorio se prepararon planos de isobatas, isohipsas, salinidad de suelos y aguas freáticas, con el fin de analizar su variación espacial y temporal, a través de interpolación por el método del Krigeado.

\section{Resultados y Discusión}

\section{Dinámica de los mantos freáticos someros}

De acuerdo con la Tabla 1 se puede observar que la variación de la profundidad de los mantos freáticos esta íntimamente ligada con la precipitación y las aplicaciones del agua de riego. Las áreas con niveles freáticos entre $0,0-0,5 \mathrm{~m}$ y $0,5-1,0 \mathrm{~m}$ se consideran críticas por el efecto adverso que pueden generar en el suelo y la planta. Los valores que se presentan aumentan a medida que aumenta la precipitación alcanzando un máximo de 52 y $36 \%$ del área total en septiembre de 2003, en el cual la precipitación fue de $346,3 \mathrm{~mm}$. En los meses posteriores cuando se presenta una disminución de la precipitación se inicia un aumento de las áreas críticas por efecto de recarga de los mantos freáticos por las aplicaciones del agua de riego, llegando a 20,8 y 33,3\% del área en el mes de abril de 2004 donde hubo una aplicación de $5.035 .261 \mathrm{~m}^{3}$ de agua para riego. Las fluctuaciones de los mantos freáticos someros en gran medida debido al efecto de las precipitaciones y a las láminas de riego indican que es muy posible la salinización de los horizontes superficiales del suelo debido a la evaporación de las aguas freáticas que tienen una alta concentración de sales solubles, afectándose como consecuencia de esto el desarrollo de los cultivos debido al nivel de agua freática cerca de las raíces de las plantas. Este hecho se ha reportado en estudios realizados por Cenicaña (1991) y Cruz (1995) en el cultivo de caña de azúcar. Las áreas de mayor intensidad de fluctuación de los mantos freáticos se localizaron en la parte central del distrito de riego La Doctrina, Córdoba, Colombia.

$\mathrm{Al}$ analizar los planos de isohipsas desde junio de 2003 hasta junio de 2004 se observó que la tendencia de la dirección del flujo subterráneo es de Este y Sureste hacia la parte Central y Noreste del distrito de riego en estudio, es decir, la zona de carga está en el Este y Sureste y la zona de descarga en la parte Central y Noreste (Figura 1).

El flujo convectivo en los distritos de riego funciona de acuerdo con la ley de Darcy; la cual describe la dirección del flujo subterráneo, con el flujo de descarga se presenta la posibilidad del transporte de sales desde la zona de carga hacia la zona de descarga del flujo subterráneo, este proceso fue encontrado por Mercado (1999) en el distrito de riego 041 río Yaqui, Sonora, México. Con respecto 
Tabla 1. Porcentaje de áreas a diferentes profundidades del nivel freático en función de la precipitación y riegos, Distrito de riego La Doctrina, Colombia

\begin{tabular}{|c|c|c|c|c|c|c|c|}
\hline \multirow[t]{2}{*}{ Mes } & \multicolumn{5}{|c|}{$\begin{array}{l}\text { Porcentaje de áreas con diferentes } \mathrm{PNF}^{1} \\
\qquad(\mathrm{~m})\end{array}$} & \multirow{2}{*}{$\begin{array}{c}\mathrm{PP}^{2} \\
(\mathrm{~mm})\end{array}$} & \multirow{2}{*}{$\begin{array}{c}\text { Riego } \\
\left(\mathrm{m}^{3}\right)\end{array}$} \\
\hline & $0-0,5$ & $0,5-1,0$ & $1,0-1,5$ & $1,5-2,0$ & $>2,0$ & & \\
\hline Junio/2003 & 28,0 & 44,0 & 12,0 & 8,0 & 8,0 & 111,9 & 0,0 \\
\hline Julio/2003 & 46,2 & 26,9 & 15,4 & 7,7 & 3,8 & 166,9 & 0,0 \\
\hline Agosto/2003 & 23,1 & 42,3 & 23,1 & 7,7 & 3,8 & 152,5 & 0,0 \\
\hline Septiembre/2003 & 52,0 & 36,0 & 12,0 & 0,0 & 0,0 & 346,3 & 0,0 \\
\hline Octubre/2003 & 15,4 & 46,2 & 30,8 & 7,7 & 0,0 & 49,1 & 0,0 \\
\hline Noviembre/2003 & 38,5 & 46,2 & 11,5 & 3,8 & 0,0 & 6,2 & 0,0 \\
\hline Diciembre/2003 & 3,8 & 46,2 & 38,5 & 11,5 & 0,0 & 147,0 & 0,0 \\
\hline Enero/2004 & 3,8 & 23,1 & 42,3 & 30,8 & 0,0 & 0,0 & 1.234 .319 \\
\hline Febrero/2004 & 11,5 & 15,4 & 30,8 & 38,5 & 3,8 & 0,0 & 4.281 .826 \\
\hline Marzo/2004 & 16,7 & 20,8 & 29,2 & 20,8 & 12,5 & 0,0 & 4.951 .157 \\
\hline Abril/2004 & 20,8 & 33,3 & 29,2 & 8,3 & 8,3 & 145,1 & 5.035 .261 \\
\hline May0/2004 & 21,7 & 30,4 & 13,0 & 30,4 & 4,3 & 190,2 & 2.565 .340 \\
\hline Junio/2004 & 20,0 & 40,0 & 32,0 & 4,0 & 4,0 & 59,4 & 1.680 .474 \\
\hline
\end{tabular}

${ }^{1} \mathrm{PNF}=$ Profundidad del nivel freático $;{ }^{2} \mathrm{PP}=$ Precipitación.

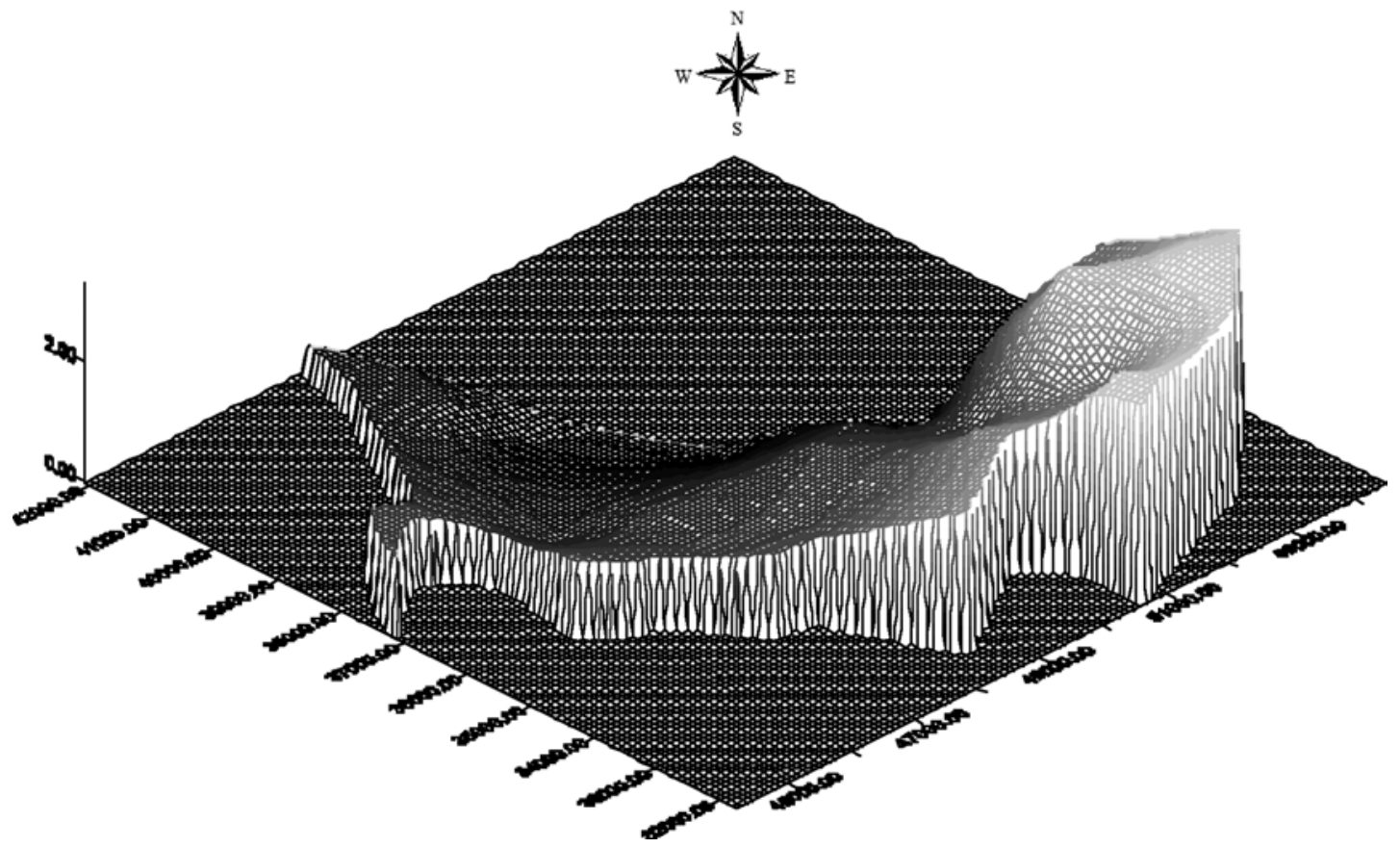

Figura 1. Plano de isohipsas, distrito de riego La Doctrina, Córdoba, Colombia, diciembre de 2003.

a la migración de sales a macroescala Hornbuckle et al. (2007); Rana et al. (2008) y Ritzema et al. (2008), consideran que los esquemas de riego para el suministro de agua a los cultivos y control de la salinidad de los suelos se debe propiciar un descenso de los niveles freáticos someros a umbrales adecuados con el fin de incrementar la producción. De allí que la operación del drenaje necesita ser optimizado de acuerdo con la dinámica de las variaciones espacio-temporales de los 
niveles freáticos, siendo muy importante conocer los planos de isohipsas.

\section{Salinidad de suelo}

De acuerdo con los resultados analíticos se observó que el pH no presentó variación espacial y temporal considerable en las épocas de los muestreos. En promedio $82,70 \%$ del área (3.440,9 hectáreas) tienen $\mathrm{pH}$ en el rango de 5-7, un $14,80 \%$ del área (615,8 hectáreas) tienen un $\mathrm{pH}$ entre 7-8, el cual puede estar asociado a la presencia de sales como el cloruro de sodio $(\mathrm{NaCl})$.

El azufre presentó valores mayores de $30 \mathrm{mg} \cdot \mathrm{Kg}^{-1}$ en un $86,2 \%$ del área en septiembre de 2003 con disminución a $23 \%$ en los otros muestreos; estos altos valores pueden generar problemas de acidez o salinidad (suelos sulfatados ácidos).

Los valores de calcio durante el muestreo presentaron valores mayores de $6 \mathrm{cmol}_{c} \cdot \mathrm{Kg}^{-1}$ en un promedio de $89,5 \%$ del área total $(3.723,8 \mathrm{ha})$; el magnesio presentó valores altos $\left(>3 \mathrm{cmol}_{c} \cdot \mathrm{Kg}^{-1}\right)$, en un promedio de $99,4 \%$ del área total (4.135,7 ha). Es de indicar que la relación calcio magnesio $\left(\mathrm{Ca}^{2+} / \mathrm{Mg}^{2+}\right)$ es menor que uno, lo cual puede causar desfloculación de la estructura del suelo, con consecuencias adversas, especialmente en lo relacionado al movimiento del agua y además forma sales de alta solubilidad. Por otra parte, el sodio presentó valores altos $\left(>1 \mathrm{cmol}_{c} \cdot \mathrm{Kg}^{-1}\right)$ en un promedio de 30,8\% del área total, con un pico máximo de 73,1\% del área total en el muestreo de marzo de 2004. Esta concentración de sodio indica que es posible ocurran problemas similares a los ocasionados por el magnesio.

En cuanto a la conductividad eléctrica del extracto de saturación del suelo (CE) se encontró que el 20,2\% del área total (840,5 ha) presentaron valores mayores de $3 \mathrm{dSm}^{-1}$, lo cual indica problemas de salinidad en estas áreas (Figura 2). En lo referente al calcio soluble en los dos primeros muestreos (septiembre y noviembre de 2003) el $24,2 \%$ del área total presentó valores mayores de $4 \mathrm{mmol}_{c} \cdot L^{-1}$, luego se presentó un descenso en el tercer muestreo (marzo 2004), posiblemente por efectos de dilución o lavados por la aplicación de riegos. Igual comportamiento presentaron el magnesio y el sodio, pero con la connotación que estos dos generan problemas considerables en el suelo.
En cuanto al bicarbonato en el primer muestreo (septiembre 2003) un 69,2\% del área total presentó valores altos $\left(>1 \mathrm{mmol}_{c} \cdot \mathrm{L}^{-1}\right)$ pero en los dos últimos muestreos (noviembre 2003 y marzo 2004) pasó a un promedio de $88,9 \%$, lo cual indica que es posible la formación de sales muy peligrosas como el carbonato de sodio $\left(\mathrm{Na}_{2} \mathrm{CO}_{3}\right)$. Los valores de sulfatos son bajos y no representan ningún riesgo. El cloruro presentó altos valores $\left(>6 \mathrm{mmol}_{c} \cdot L^{-1}\right)$ en los dos primeros muestreos, con un promedio de $28 \%$ del área total, lo cual hace factible la formación de sales de cloruro, especialmente con el sodio y magnesio.

Esta dinámica de sales en el suelo muestra la aplicabilidad de los resultados de los trabajos realizados por Degioanni et al. (2006) en zonas de irrigación en Córdoba, Argentina, en la cual usando el modelo de simulación FREAT1 realizaron excelentes predicciones de las oscilaciones del manto freático y del riesgo de salinidad de los suelos.

\section{Salinidad de aguas freáticas}

$\mathrm{El} \mathrm{pH}$ en el primer muestreo (septiembre de 2003) se encontró que el 57,7\% del área total las soluciones freáticas estaban en el rango de 7-8 y posteriormente en los dos muestreos adicionales pasaron a un promedio de $88,5 \%$, es de anotar que este rango es característico de sales como el cloruro de sodio $(\mathrm{NaCl})$. La conductividad eléctrica presentó valores altos $\left(>3 \mathrm{dSm}^{-1}\right)$ en un promedio de $21,6 \%$ del área total (Figura 3), coincidiendo geográficamente con el área de $\mathrm{pH}$ en el rango de 7-8, lo que evidencia la presencia de sales altamente solubles y móviles como el cloruro de sodio. Es importante anotar que las variaciones de la conductividad eléctrica se deben a la dilución de las soluciones freáticas por las lluvias o riegos en el área de estudio. En cuanto a los valores de calcio no presentan riesgos. Pero con el magnesio el 20,2\% del área total presentó valores altos $\left(>8 \mathrm{mmol}_{c} \cdot L^{-1}\right)$, lo cual genera un alto riesgo de formación de sales magnésicas, las cuales son muy peligrosas en el suelo porque causan desfloculación limitando el movimiento de agua. El sodio presentó altos valores $\left(>30 \mathrm{mmol}_{c} \cdot L^{-1}\right)$ en el primer muestreo con un 15,3\% del área total 


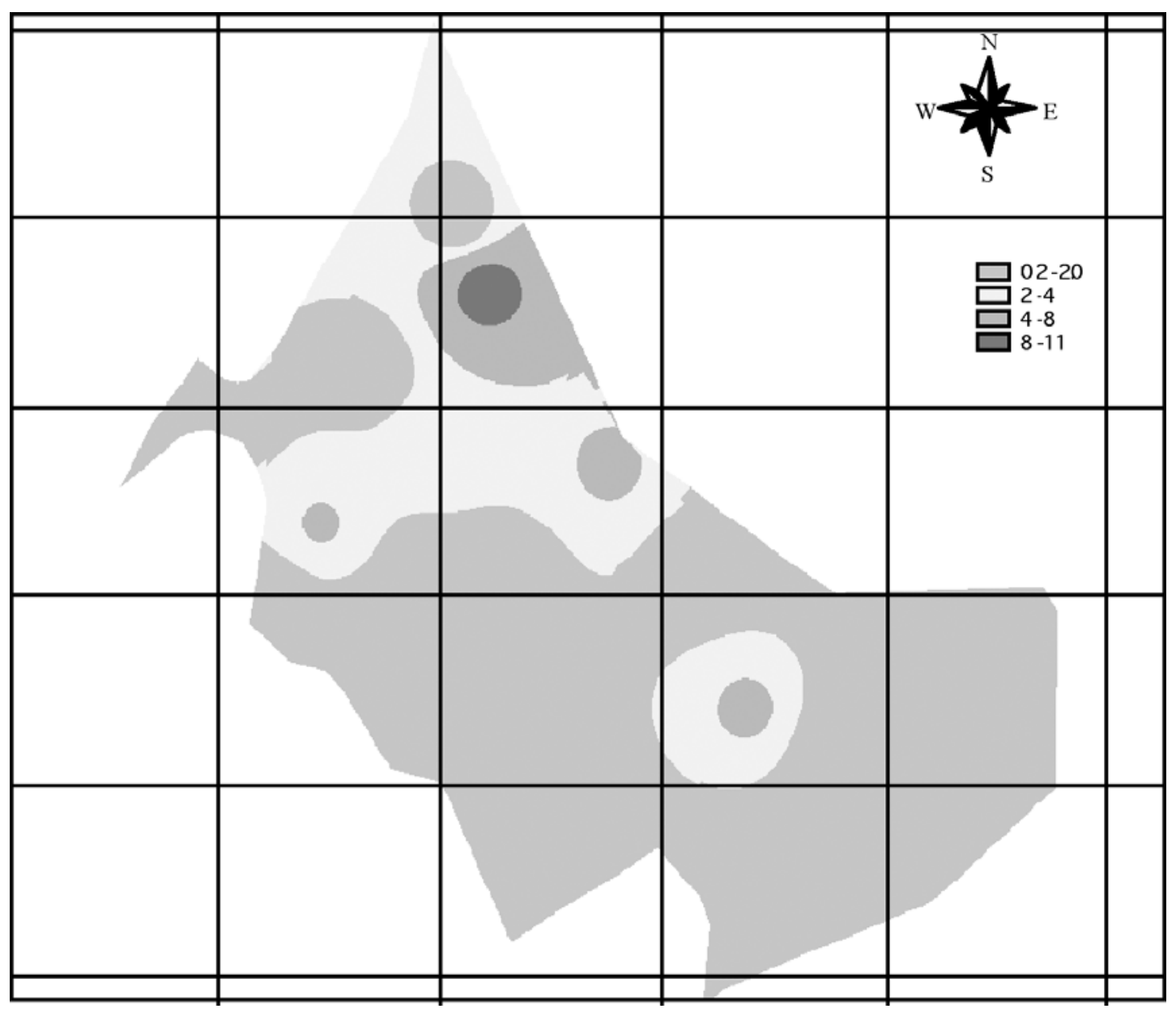

Figura 2. Plano de conductividad eléctrica del extracto de saturación del suelo (dS/m), distrito de riego La Doctrina, Colombia, marzo de 2004.

y posteriormente bajó a $8,3 \%$ posiblemente por efectos de dilución; es importante señalar que las sales de sodio tienen los mismos efectos que las de magnesio. En lo concerniente a carbonato $\left(\mathrm{CO}_{3}\right)$ el 36,9\% del área total presentó valores medios $\left(1-3 \mathrm{mmol}_{c} \cdot \mathrm{L}^{-1}\right)$, generando sales de carbonato, como el carbonato de sodio. Con el bicarbonato $\left(\mathrm{HCO}_{3}\right)$ se encontró que el 93,6\% del área total presentó valores altos $\left(>1 \mathrm{mmol}_{c} \cdot L^{-1}\right)$, lo cual hace posible la presencia de sales de bicarbonato. El cloruro $(C l)$ el 19,5\% del área presentó valores altos $\left(>20 \mathrm{mmol}_{c} \cdot L^{-1}\right)$ lo cual hace factible la presencia de sales muy solubles como el cloruro de sodio y el cloruro de magnesio.
Se encontró que las zonas de mayor concentración de sales coinciden con las zonas de descarga del flujo freático, lo cual indica que el transporte de sales ocurre en la dirección del flujo subterráneo o flujo Darcyniano (flujo convectivo), este resultado coincide con lo reportado por Mercado (1999) en el distrito de riego 041 río Yaqui, Sonora, México; y por Ortiz et al. (2005) en áreas irrigadas de la provincia de Mendoza, Argentina.

\section{Conclusiones}

1. El cloruro de sodio y magnesio son las principales sales que predominan en el suelo y las aguas freática del distrito de riego y drenaje La Doctrina, Córdoba, Colombia.

2. Existe un alto porcentaje del área de estudio con conductividad eléctrica del agua freática 


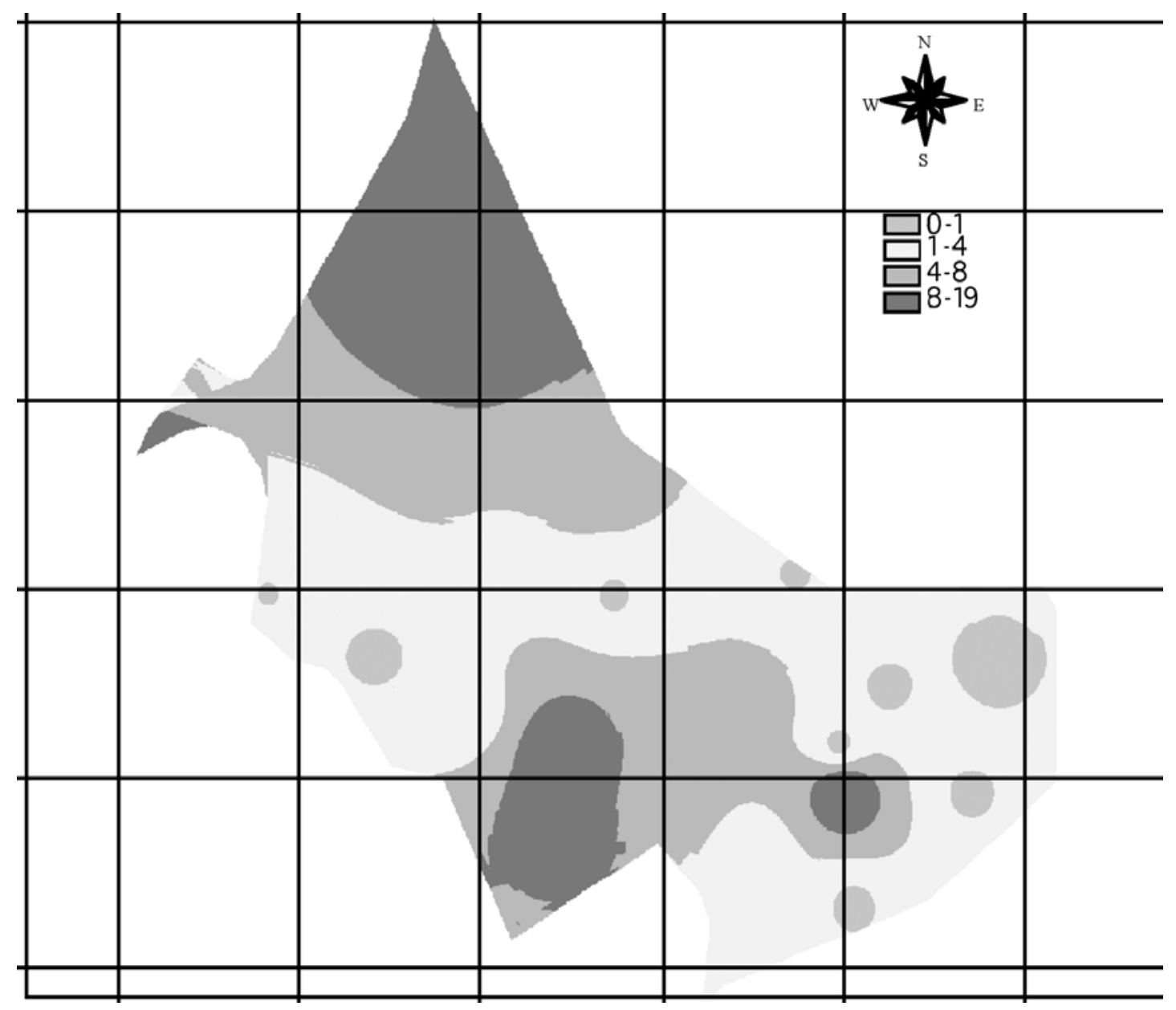

Figura 3. Plano de conductividad eléctrica $(\mathrm{dS} / \mathrm{m})$ de aguas freáticas, distrito de riego La Doctrina, Córdoba, Colombia, marzo de 2004.

mayor de $3 \mathrm{dSm}^{-1}$ y profundidad de los mantos freáticos menor de $1,0 \mathrm{~m}$, lo cual representa alto riesgo de salinidad para el suelo.

3. La variación de la distribución espacial de la salinidad del suelo y las aguas freáticas se debe a los procesos de dilución por la precipitación y los riegos.

4. La migración de sales se da en la dirección del flujo freático, lo cual es responsable de la salinización progresiva del suelo.

\section{Literatura Citada}

American Public Health Association (Apha) 1995 Standard methods for examination of water and wastewater. $19^{\text {th }}$ Edition. Washington DC. 380 p.

Arenas, A.; Aduen, H.; Mercado, T.

2003 Estudio de los niveles freáticos en el distrito de riego y drenaje de La Doctrina, Córdoba, Colombia. Ediciones Universidad de Córdoba. Montería. 60 p.

Bustamante, $\mathrm{H}$.

1993 Drenaje de tierras agrícolas y sus aplicaciones. Ediciones Universidad Nacional de Colombia, sede Medellín. Medellín, Colombia. 247 p.
Centro de Investigaciones de La Caña de Azúcar de Colombia (Cenicaña)

1991 Efecto del nivel freático en la producción de caña de azúcar. Informe Anual CENICAÑA, Cali, Colombia: 32-33.

Comisión Nacional del Agua (CNA)

2007 Manejo del régimen de humedad en suelos del trópico húmedo de México. CNA. México. 19 p.

Cruz, R.

1995 Drenajes en el cultivo de la caña en la zona azucarera de Colombia. Cenicaña, Cali, Colombia: 211-233. 
Custodio, E; Llamas, M.R.

1983 Hidrología subterránea. Tomo I. Ediciones Omega, S.A. Barcelona. 1157 p.

Degioanni, A.; Cisnero, J.; Cantero, A.; Videla, H.

2006 Modelo de simulación del balance hídrico en suelos con agua freática poco profunda. Revista Sociedad Argentina de la Ciencia del Suelo 1: 1-10.

Espinoza, R.M.

1995 Estudio del comportamiento de los niveles freáticos con respecto al bombeo en el valle del Yaqui, Sonora, México. Tesis de licenciatura, Hermosillo, México. Instituto Tecnológico de Sonora (ITSON), Sonora, México. 100 p.

Hassan, E.

1994 Balance de agua y de sales en un "Gypsic Xerochrept" situado en la cuenca media del río Guadiana. Tesis doctorado, Madrid, España. Escuela Técnica Superior de Ingenieros Agrónomos. Universidad Politécnica de Madrid, España. $180 \mathrm{p}$.

Hornbuckle, J.W.; Christen, E.W.; Faulkner, R.D.

2007 Evaluating a multi-level subsurface drainage system for improved drainage water quality. Agricultural Water Management: 208-216.

Lázaro, P.; Fuentes, C.; Collado, J.; De Leon, B.

2005 Modelación inversa de la recarga de los mantos freáticos someros en distritos de riego. Revista Ingeniería Hidráulica en México, Vol. XX, № 1, enero-marzo: 89-98.

Martínez, J.

1986 Drenaje agrícola. Volumen I. Edición Ministerio de agricultura, pesca y alimentación. Madrid. 140 p.

Mercado, T.

1999 Establecimiento de vectores de salinidad a través del análisis físico-químico de los mantos freáticos someros en el distrito de riego 041 río Yaqui, Sonora, México. Tesis maestría, Montecillos, México. Centro de Hidrociencias, Colegio de Postgraduados, Montecillo, México. 135 p.

Niazi, M.F.K.; Ghumman, A.R, Wolters, W.

2008 Evaluation of impact of Khushab subsurface pipe drainage project in Pakistan. Irrigation and Drainage System: $35-45$.

Ortiz, G.; Morábito, J.; Rearte, E.; Mastrantonio, L.

2005 Salinidad del agua freática en el área regadía del río Mendoza. Revista de la Facultad de Ciencias Agrarias, Universidad de Mendoza, Argentina: 1-14.

Pizarro, F.

1978 Drenaje agrícola y recuperación de suelos salinos. Editorial Agrícola Española: Madrid. 203 p.

Rana, T.; Khan, S.; Rahimi, M.

2008 Spatio-temporal optimization of agricultural drainage using groundwater models and genetic algorithms: An example from the Murray Irrigation Area, Australia. Hydrogeology Journal: 1145-1157.

Ritzema, H.; Satyanarayana, T.; Raman, S.; Boonstra, J.

2008 Subsurface drainage to combat waterlogging and salinity in irrigated lands in Índia: Lessons learned in farmers' fields. Agricultural Water Management: 179-189.

Tanji, K.

1990 Agricultural salinity assessment and management. Published by ASCE: New York. 619 p.

Timm, L.; De Oliveira, J.; Tominaga, T.; Cássaro, F.; Reichardt, K.; Bacci, O.

2002 Water balance of a sugarcane crop: Quantitative and qualitative aspects of its measurement. Revista Brasileira de Engenharia Agrícola e Ambiental (6): 57-62.

Yang, $\mathrm{X}$.

2008 Evaluation and application of DRAINMOD in Australian sugarcane field. Agricultural Water Management: 439-446. 